\title{
An italic obsession: electoral reforms
}

Citation: A. Chiaramonte (2020) An italic obsession: electoral reforms. Quaderni dell'Osservatorio elettorale - Italian Journal of Electoral Studies 83(1): 5-16. doi: 10.36253/qoe-9528

Received: February 21, 2020

Accepted: May 8, 2020

Published: July 28, 2020

Copyright: (c) 2020 A. Chiaramonte. This is an open access, peer-reviewed article published by Firenze University Press (http://www.fupress.com/qoe) and distributed under the terms of the Creative Commons Attribution License, which permits unrestricted use, distribution, and reproduction in any medium, provided the original author and source are credited.

Data Availability Statement: All relevant data are within the paper and its Supporting Information files.

Competing Interests: The Author(s) declare(s) no conflict of interest.

\author{
Alessandro Chiaramonte \\ Department of Political and Social Sciences, University of Florence, Italy \\ E-mail: alessandro.chiaramonte@unifi.it
}

\begin{abstract}
The history of Italy is plenty of reforms of the electoral system. Many are those implemented since the country's unification: from the majority system to the limited vote, from proportional representation to the majority premium in the liberal era; and, again, in the Republican era, the return to proportional representation and then the use of mixed systems, combining PR with plurality or majority premium. And many other are the reforms which, discussed and sometimes even approved, as in the case of the italicum, have remained dead letter or never saw the light. What explains this Italic obsession with the electoral systems? Why have their reforms been on the parties' and governments' political agenda for so long? The goal of this article is to answer these questions. In the end, electoral reforms have played as instruments of coordination and adaptation in the political strategies pursued by the parties in specific time periods and also as substitute instruments of institutional engineering in the absence of broader agreements on major constitutional reforms.
\end{abstract}

Keywords. Electoral reforms, electoral engineering, Second Republic, Italy.

\section{INTRODUZIONE: \\ LE RIFORME ELETTORALI ITALIANE IN PROSPETTIVA STORICA}

Dall'unità ad oggi, la storia d'Italia è costellata di numerose riforme elettorali, molte più di quelle che hanno avuto luogo nello stesso arco di tempo in altri paesi dell'Europa occidentale, la Gran Bretagna su tutti, ma non solo. In estrema sintesi, in epoca liberale si è passati dal maggioritario di collegio allo scrutinio di lista, e poi alla proporzionale e, con l'avvento del fascismo, al premio di maggioranza; e, ancora, in epoca repubblicana, si è assistito al ritorno alla proporzionale e poi al ricorso a vari sistemi misti, in cui alla proporzionale si sono aggiunti nuovamente prima i collegi uninominali, poi il premio di maggioranza, e poi, di nuovo, i collegi uninominali. Oltre alle tante - grandi e piccole - riforme elettorali attuate, non pochi sono stati poi i tentativi di riforma di cui si è ampiamente discusso ma che infine non sono andati in porto; in taluni casi, come per il cosiddetto italicum, essi hanno persino superato l'approvazione parlamentare ma non il vaglio (complessivo) della Corte costituzionale.

La riforma del sistema elettorale non è dunque mai del tutto scomparsa dall'agenda politica italiana. Tuttavia, ci sono state fasi storiche diver- 
se tra loro quanto a intensità e frequenza del dibattito. E la fase che più di ogni altra è stata caratterizzata da una sequenza relativamente ravvicinata di riforme elettorali attuate (ben sei, come vedremo) e tentate (almeno altrettante) è quella degli ultimi trent'anni, coincidente con la crisi della (cosiddetta) Prima Repubblica e con la transizione alla e affermazione della (cosiddetta) Seconda Repubblica. Su questa fase ci concentreremo qui, per cercare di spiegare questa ossessione italiana per i sistemi elettorali. Nel farlo circoscriveremo l'analisi ai sistemi di elezione del parlamento nazionale, sebbene in taluni casi faremo comunque riferimento anche alle riforme che hanno avuto luogo ai livelli di governo sub-statale. L'obbiettivo è di mettere in luce le motivazioni di questa continua rincorsa ad un sistema elettorale «migliore» - 0 , forse, più semplicemente, più «adatto» alle mutate condizioni - a scapito della loro stabilità, che - ricordiamolo - è a sua volta necessaria a fornire stabilità al sistema politico nel suo complesso. Una rincorsa caratterizzata, oltretutto, dalla compresenza di attori (quali gli elettori che si sono espressi per via referendaria e i giudici della Corte costituzionale che si sono espressi con le loro sentenze) che hanno seriamente sfidato il primato del parlamento (e in esso dei partiti) su questa materia.

\section{DAL PROPORZIONALE AL "MAGGIORITARIO»: LA RIFORMA DEL 1993}

La Prima Repubblica è stata la «Repubblica della proporzionale». Dato il contesto politico-ideologico non avrebbe potuto essere diversamente, anche se ai tempi dell'Assemblea costituente c'era chi sosteneva l'adozione di un sistema maggioritario e ancora nel 1953 De Gasperi tentò la via di una correzione «forte» al sistema proporzionale grazie al premio di maggioranza (Piretti 2003; Quagliarello 2003). Alla lunga, però, il sistema proporzionale "puro» comincia ad essere ritenuto corresponsabile degli evidenti problemi di inefficacia del governo e di mancanza di ricambio della classe politica. Non sorprende dunque che negli anni ' 80 vengano avanzate e discusse alcune proposte di riforma elettorale (Pasquino 1982; 1985; Ruffilli 1987), anche se si trattava per lo più di correttivi al sistema proporzionale in vigore, che sembrava ancora inattaccabile nelle sue fondamenta. Di lì a poco, però, tutto sarebbe cambiato, grazie soprattutto allo strumento del referendum abrogativo (Giannetti e Grofman 2011; Renwick 2010; Uleri 2003). La prima riforma attuata fu ancora interna al vecchio sistema elettorale. Nel 1991 il numero dei voti di preferenza fu ridotto a uno ${ }^{1}$.

${ }^{1}$ In precedenza l'elettore poteva esprimere, a seconda dell'ampiezza della circoscrizione di residenza, fino ad un massimo di 2 (piccole
La modifica fu considerata un successo del movimento referendario che l'aveva promossa, ma le sue conseguenze non furono del tutto positive (Pasquino 1993). Di ben altra portata la riforma che scaturì dal referendum sulla legge elettorale del Senato che si svolse il 18 Aprile 1993 e che fu approvato a larghissima maggioranza $(82,7 \%$ di si) con una affluenza alle urne pari al $77,1 \%$ degli aventi diritto. L'esito sbloccò l'impasse sulla riforma elettorale e demolì le resistenze dei difensori della proporzionale. Dopo un passaggio parlamentare comunque contrastato (Pappalardo 1995), fu approvata la legge 276/1993 che venne battezzata «legge Mattarella». Pur con alcune modifiche di una certa importanza, essa ricalcava il mix di maggioritario e proporzionale uscito dal referendum e prevedeva dunque un sistema elettorale misto in cui il $75 \%$ di seggi veniva assegnato in collegi uninominali a turno unico e il restante $25 \%$ con formula proporzionale.

A parte questa caratteristica comune, i sistemi elettorali di Camera e Senato differivano tra loro per alcuni aspetti non del tutto secondari, forieri - e in effetti così sarebbe avvenuto ${ }^{2}-$ di esiti diversi: 1) alla Camera gli elettori disponevano di due schede distinte e dunque potevano votare separatamente per i candidati di collegio e per le liste concorrenti nella parte proporzionale, al Senato vi era invece un'unica scheda e il voto valeva contestualmente per candidati e liste (qui chiamate gruppi di candidati); 2) la formula per la ripartizione dei seggi proporzionali era il quoziente naturale e i più alti resti applicato in un'unica circoscrizione nazionale alla Camera, era invece il d'Hondt applicato in circoscrizioni coincidenti con le regioni al Senato; 3) la soglia di sbarramento era fissata al $4 \%$ a livello nazionale alla Camera, mentre non c'era al Senato, dove però la combinazione di metodo d'Hondt e circoscrizioni con un numero relativamente basso di seggi proporzionali da attribuire determinava una soglia di fatto variabile ma generalmente piuttosto alta, superiore a quella della Camera; 4) lo scorporo, ossia la quota di voti sottratta alle liste concorrenti

circoscrizioni), ovvero di 3 (medie), ovvero di 4 (grandi) voti di preferenza. All'epoca del referendum, e sulla base di un filone consolidato di studi (Cazzola 1972; D’Amico 1987; Katz e Bardi 1979; Pasquino 1972), il voto di preferenza multiplo era considerato un fattore in grado di alimentare il frazionismo interno ai partiti (attraverso le cosiddette "cordate») e il voto clientelare.

${ }^{2}$ Nelle elezioni del 1994 le forze di centro-destra - a prescindere dalle diverse soluzioni coalizionali adottate nelle varie aree territoriali del paese - ottennero la maggioranza assoluta dei seggi alla Camera ma non al Senato. A parti invertite, nel 1996 l'Ulivo consegui una maggioranza autosufficiente al Senato, ma non alla Camera. Del resto, un sistema prevalentemente maggioritario come quello della legge Mattarella, a causa dell'effetto leva del collegio uninominale (combinato alle differenze sopra descritte tra le due camere, oltre che a quella dei rispettivi corpi elettorali), ben poteva amplificare anche piccole differenze di risultati tra Camera e Senato, con il rischio di esiti diversi. 
nella parte proporzionale per ogni candidato di collegio ad esse collegato e risultato vincente, era «totale» (sottrazione di tutti i voti ottenuti dai candidati di collegio vittoriosi) al Senato e "parziale» alla Camera (sottrazione dei soli voti sufficienti a consentire la vittoria dei candidati di collegio, pari ai voti dei candidati secondi arrivati aumentati di una unità) (D'Alimonte e Chiaramonte 1995).

La riforma elettorale del 1993 non fu dunque il prodotto di un processo di ingegneria istituzionale avviato dalle forze politiche (Donovan 1995). Al contrario, fu imposta dall'esito del referendum sulla legge elettorale del Senato che fu promosso contro la volontà di gran parte della classe politica di allora. Il successo dell'iniziativa referendaria, determinato dall'insoddisfazione dell'elettorato nei confronti del sistema politico ma anche dalla volontà di dare una scossa al processo di mutamento istituzionale, rappresentò un vincolo per la classe politica che approvò la nuova complessiva legislazione elettorale. In altri termini, la riforma elettorale del $1993 \mathrm{fu}$ il prodotto di una decisione combinata tra elettori e classe politica (Renwick 2010).

Sorretta dalla retorica del «maggioritario», che avrebbe accompagnato costantemente la transizione dalla Prima alla Seconda Repubblica e che si sarebbe prolungata ben oltre, la riforma del 1993 fu concepita da molti come il «grimaldello» per scardinare l'assetto istituzionale fino ad allora esistente, così da creare le condizioni di una democrazia che, per usare i termini di Lijphart (2012), fosse meno «consensuale»e, appunto, più «maggioritaria», quantomeno con riferimento alla «dimensione esecutivo-partiti». Una democrazia competitiva e decidente che non poteva essere lasciata però all'azione del solo sistema elettorale, ma che andava invece assecondata con opportune riforme costituzionali.

Le aspettative di molti erano che i nuovi sistemi elettorali di Camera e Senato avrebbero ridotto il numero dei partiti e attenuato la rispettiva distanza ideologica, favorendo così l'affermazione di un sistema partitico più competitivo. Gli elettori avrebbero potuto scegliere tra differenti alternative partitiche candidate al governo del paese e finalmente - considerata la storia della Prima Repubblica - ci sarebbe stata un'effettiva possibilità di alternanza di governo.

Solo alcune di queste aspettative si sono realizzate. Da un lato, i nuovi sistemi elettorali quasi-maggioritari hanno certamente contribuito all'affermarsi di una competizione bipolare, ossia caratterizzata (tendenzialmente) dalla presenza di due coalizioni ampie percepite dagli elettori come effettivi sfidanti per la conquista della maggioranza assoluta dei seggi. In questo contesto gli elettori hanno avuto un ruolo molto più importante che in passato, potendo non solo votare per il partito preferito ma scegliendo, di fatto, anche il governo (o, meglio, la coalizione al governo) e finanche il capo di governo (il leader della coalizione vincente). Ciò ha reso possibile anche l'alternanza al governo tra schieramenti politici opposti, favorendo al contempo un rapporto di maggiore responsabilità (accountability) degli eletti nei confronti degli elettori. Dall'altro lato, l'attesa riduzione del numero dei partiti non solo non si è avverata, ma si è registrato addirittura un significativo aumento. Non a caso il sistema partitico italiano emerso dalla riforma elettorale del 1993 - e dalle ceneri del vecchio sistema di pluralismo polarizzato (Sartori 1976) - è stato definito come un «bipolarismo frammentato» (D’Alimonte 2005).

La mancata riduzione, ed anzi l'esplosione, della frammentazione partitica trova le sue radici nella destrutturazione del sistema partitico italiano in coincidenza con la fase in cui le nuove regole elettorali vennero introdotte. Molti dei partiti della Prima Repubblica erano stati ridimensionati, o si erano divisi, o erano addirittura scomparsi. Nuovi partiti si erano costituiti ed erano alla ricerca di quella visibilità che avrebbe consentito loro la sopravvivenza e, possibilmente, l'affermazione nelle mutate condizioni. In ogni caso era chiaro che nessuno di loro, nuovo o vecchio che fosse, sarebbe stato sufficientemente forte da poter presentare da solo i propri candidati nei collegi uninominali potendo ambire a vincerli. Al contrario, dato il contesto competitivo che si stava sviluppando in virtù dei nuovi sistemi elettorali quasi-maggioritari e l'intento generalizzato di perseguire benefici elettorali nel breve periodo, era chiaro che le speranze di vittoria nei collegi uninominali sarebbero dipese dalla definizione di alleanze tra partiti, possibilmente (ma non necessariamente) affini dal punto di vista ideologico e/o programmatico, a sostegno di candidati comuni. È così, sulla base degli incentivi posti dal collegio e nel quadro di un sistema partitico destrutturato, che nacquero le coalizioni (pre-elettorali) che sarebbero divenute i protagonisti della sfida del voto. Tuttavia, le condizioni affinché l'alleanza tra partiti prendesse forma furono particolarmente complesse, poiché dovevano tener conto che ciascuno dei partner pretendeva per $\mathrm{i}$ propri affiliati una quota dei candidati di collegio comuni - una quota commisurata al contributo in voti che il partito si presupponeva portasse in dote alla coalizione, secondo una logica di "proporzionalizzazione» del maggioritario (D'Alimonte 2001) - oltre che la collocazione almeno di una parte di costoro in collegi ove la coalizione avesse possibilità se non certezza di vittoria (Di Virgilio 2002). Insomma, il processo di coalition-building risultava davvero complicato e in ogni caso si concludeva con ampie concessioni nei confronti dei partiti mino- 
ri (in termini di candidature di collegio, ma anche di promesse di cariche di governo e di sotto-governo), dei quali i partiti maggiori temevano le minacce di defezione e il relativo costo (che poteva ben essere la sconfitta della coalizione nella corsa al governo). In tal modo la frammentazione nata dal processo di destrutturazione del sistema partitico della Prima Repubblica trovava linfa vitale per perpetuarsi e, persino, per proliferare.

Oltretutto, la frammentazione partitica si trasferiva dal livello elettorale a quello parlamentare e infine a quello governativo, con inevitabili conseguenze negative sulla coesione programmatica e sulla stabilità degli esecutivi. In altri termini, l'affermazione di un sistema partitico bipolare fondato su coalizioni catch-all si accompagnava ad un vero e proprio paradosso: una coalizione quanto più ampia e inclusiva possibile (così da sommare i voti dei partiti componenti) era necessaria per vincere le elezioni, ma, una volta vinte le elezioni, si rivelava un ostacolo quasi insormontabile per fornire un indirizzo politico unitario e, dunque, per governare con efficacia il paese ${ }^{3}$.

È su quest'ultimo aspetto che si sono appuntate gran parte delle critiche alla riforma elettorale del 1993. Sartori ne parlò in termini dispregiativi etichettando il nuovo sistema come mattarellum e mantenendo questo giudizio sino in fondo (Sartori 2001), in linea con il suo giudizio negativo sul genere dei sistemi elettorali misti, definiti dei «bastardi» rispetto ai sistemi proporzionali e maggioritari (Sartori 1995). Coloro che ritenevano la componente proporzionale del sistema la causa dell'enorme frammentazione partitica si spinsero fino al punto di richiederne l'abolizione per via referendaria. Furono fatti due tentativi nel 1999 e nel 2000. Entrambi fallirono per il mancato raggiungimento del quorum di votanti. Sta di fatto che, dopo tre elezioni, e senza particolari resistenze, la legge Mattarella fu superata. Ma paradossalmente nel nuovo sistema a rimanere, ed anzi ad espandersi, fu la parte proporzionale, mentre la parte dei collegi uninominali sparì a favore dell'introduzione del premio di maggioranza.

A dispetto delle tante critiche subite, vale la pena ricordare però che la legge Matterella aveva comunque contribuito in maniera decisiva alla trasformazione

\footnotetext{
${ }^{3}$ Il giudizio negativo sullefficacia di governo in epoca «maggioritaria» va comunque commisurato alle aspettative che al tempo della riforma elettorale del 1993 erano addirittura di governi in grado di durare per l'intera legislatura. Da una diversa prospettiva, limitandosi a considerare la stabilità dei governi nei termini della loro durata in confronto a quelli della prima Repubblica, allora le cose cambiano. Se nel periodo 1948-1994 la durata media dei governi è stata di 322 giorni, nel periodo 1994-2006 (quello corrispondente alle legislature inaugurate con elezioni in cui era vigente la legge Mattarella) la durata media dei governi è stata di 523 giorni. In seguito, dopo la riforma elettorale del 2005, e fino alle elezioni del 2013, tale durata sarebbe ulteriormente aumentata.
}

della meccanica del sistema partitico in senso bipolare, rendendo gli elettori arbitri della scelta dei governi e favorendo l'alternanza al potere - risultati conformi alle aspettative del movimento referendario della prima metà degli anni '90. E se non tutto era andato per il verso atteso, lo si doveva alla circostanza che il sistema elettorale aveva agito in un contesto istituzionale che era lo stesso della Repubblica del proporzionale (con i relativi incentivi alla frammentazione partitica) e che la riforma costituzionale alla quale si doveva accompagnare era rimasta solo un'intenzione.

\section{IL PREMIO DI MAGGIORANZA: LA RIFORMA DEL 2005}

Nel dicembre 2005, a pochi mesi dalla fine della legislatura, la coalizione di centro-destra allora maggioritaria in parlamento approvò una nuova riforma elettorale (legge Calderoli, 270/2005) con l'intento di prevenire ovvero di minimizzare la sconfitta che i sondaggi gli pronosticavano nelle future elezioni politiche (Chiaramonte and Di Virgilio 2006; Pasquino 2007). Diversamente da quella del 1993, la riforma del 2005 fu imposta dunque dall'élite politica (Renwick 2010; Baldini 2011), anche se in modo non consensuale.

Specificamente, l'obbiettivo della riforma era l'eliminazione del collegio uninominale, con il quale il centrodestra si trovava in una situazione di svantaggio competitivo nei confronti del centro-sinistra (ossia i candidati di collegio del centro-destra ottenevano sistematicamente meno voti delle liste proporzionali che li appoggiavano, mentre per i candidati di collegio di centrosinistra avveniva esattamente l'opposto) (Chiaramonte e D'Alimonte 2006; Chiaramonte e Di Virgilio 2006) ed era costretto a passare attraverso estenuanti negoziazioni intra-coalizionali per definire le candidature comuni (Renwick, Hanretty e Hine 2009). Ne scaturì l'adozione di un sistema elettorale ancora una volta misto, ma del tipo proporzionale a premio di maggioranza.

Le legge Calderoli prevedeva alla Camera dei deputati una prima distribuzione proporzionale dei seggi a livello nazionale tra le liste e le coalizioni di liste - identificate da un "capo» e da un programma comuni - che avevano superato le soglie di sbarramento (rispettivamente $4 \%$ e $10 \%$ ). Qualora la lista o coalizione di liste con il maggior numero di voti non avesse ottenuto almeno 340 seggi (pari a circa il $54 \%$ per cento di quelli totali), allora sarebbe scattato il premio di maggioranza, così da consentire il raggiungimento dei 340 seggi. In questo caso le liste e le coalizioni perdenti si sarebbero ripartiti proporzionalmente i seggi rimanenti. Le modalità di 
assegnazione del premio di maggioranza rendevano questo sistema elettorale majority-assuring, ossia in grado di determinare in ogni caso l'esito di una lista o coalizione destinataria della maggioranza assoluta dei seggi.

Il sistema elettorale del Senato seguiva l'impianto di quello della Camera, ma ancora una volta con significative differenze. In ossequio ad una certa interpretazione (allora prevalente) dell'art. 57 della Costituzione ${ }^{4}$, il premio di maggioranza non veniva assegnato a livello nazionale, bensì a livello regionale in 17 regioni su $20^{5}$. In queste 17 regioni la lista o la coalizione di liste che avesse ottenuto un voto più degli altri avrebbero incassato un premio pari al 55\% dei seggi spettanti a quella regione. Le liste e le coalizioni perdenti si sarebbero ripartiti proporzionalmente i seggi rimanenti, purché avessero superato le soglie di sbarramento (rispettivamente $8 \%$ e $20 \%$ dei voti regionali). Diversamente dalla Camera dei deputati, al Senato il premio di maggioranza (che, a questo, punto non si può neppure chiamare tale) non garantiva la determinazione di un vincitore con la maggioranza assoluta dei seggi ${ }^{6}$ (Chiaramonte e D’Alimonte 2006).

Il premio di maggioranza non era una novità per l'Italia. Non solo perché, come abbiamo già accennato,

\footnotetext{
${ }^{4}$ Lart. 57 della Costituzione recita che il «Senato della Repubblica è eletto a base regionale». Ciò è stato a lungo - e ancora al tempo della riforma del 2005 - inteso come un limite invalicabile all'adozione nella legge elettorale di qualsiasi meccanismo (ad esempio il premio di maggioranza o una soglia di sbarramento, se applicati nazionalmente) che agisse ad un livello sovraordinato a quello regionale. Questa interpretazione dell'art. 57 è stata peraltro successivamente superata, a favore di una interpretazione meno estesa che ritiene inderogabile esclusivamente l'integrità numerica della rappresentanza di ciascuna regione, ossia il numero di seggi spettanti a ciascuna di esse in base alle norme vigenti. Ė alla luce di questi sviluppi dottrinari che, nella legge Rosato del 2017, si è potuto introdurre anche per l'elezione del Senato una soglia di sbarramento nazionale. Sul punto si veda Tarli Barbieri (2018, 33-35).

${ }^{5}$ Le tre regioni nelle quali non si assegnava alcun premio erano il Molise, la Valle d'Aosta e il Trentino-Alto Adige. In Molise i due seggi in palio erano ripartiti con formula proporzionale. La Valle d'Aosta era costituita in collegio uninominale per l'attribuzione al candidato più votato dell'unico seggio ad essa spettante. Il Trentino-Alto Adige consisteva di sei collegi uninominali assegnati con il maggioritario e di un settimo seggio attribuito proporzionalmente e con scorporo totale (come nella vecchia legge Mattarella). Infine, i seggi della circoscrizione estero venivano suddivisi proporzionalmente nell'ambito di quattro distinte ripartizioni geografiche.

${ }^{6}$ Anche nel caso della legge Calderoli - come e ancor più che nel caso della legge Mattarella - le differenze tra i sistemi elettorali di Camera e Senato erano foriere di esiti diversificati. A questo proposito basti pensare al risultato delle elezioni del 2013, quando la coalizione di centro-sinistra ottenne circa il 55\% dei seggi alla Camera e meno del $40 \%$ dei seggi al Senato (dove peraltro aveva ricevuto una più alta percentuale di voti). Meno eclatante, ma pur sempre indicativo, anche l'esito delle elezioni del 2006 che vide l'Unione di Prodi conquistare una maggioranza di seggi ampia alla Camera (55\%) e risicatissima al Senato (appena sopra il 50\%)
}

aveva trovato applicazione anche nel passato, nel 1953 (legge De Gasperi) e, precedentemente, nelle elezioni del 1924 (legge Acerbo). Ma soprattutto perché già da prima del 2005 era vigente a livello comunale e provinciale (specificamente dal 1993) e regionale (dal 1995), dove si associava all'elezione diretta del capo dell'esecutivo (che dunque godeva di una maggioranza consiliare fabbricata dal sistema elettorale) ${ }^{7}$ (Chiaramonte e Tarli Barbieri 2011). Va rilevato come, dal punto di vista della coerenza dei sistemi elettorali tra i vari livelli di governo, per oltre un decennio la legge Mattarella aveva costituito un'anomalia, mentre la legge Calderoli ne rappresentava il compimento. Oltretutto, la riforma elettorale del 2005 - con la sua quasi-investitura diretta del governo - assecondava di fatto il disegno di rafforzamento dell'esecutivo nazionale previsto dalla legge di revisione costituzionale approvata nello stesso anno dal parlamento (e che sarebbe stata poi rigettata per via referendaria).

Sin dalla sua prima applicazione, la legge Calderoli è stata aspramente criticata (Di Virgilio 2007a; 2007b; Pasquino 2007), anche se non da tutti (Feltrin e Fabrizio 2007; 2008). Sartori l'avrebbe ribattezzata porcellum, a sottolinearne i difetti. Tuttavia, nelle elezioni del 2006 essa non modificò la tendenza al bipolarismo frammentato avviata dalla legge Mattarella. Nel 2008 sembrò addirittura che il formato del sistema partitico potesse finalmente ridursi, grazie all'affermazione di due grandi partiti come il Pdl e il Pd. Ma si trattò di un fuoco di paglia. Nel 2013 però - ed è storia recente - sono emersi in tutta evidenza i limiti di due sistemi elettorali mal congegnati.

Il primo limite riguardava le differenze tra i sistemi elettorali di Camera e Senato e, dunque, i rischi di esiti diversi tra i due rami del parlamento. In particolare, il sistema elettorale del Senato, combinando 17 premi regionali, si configurava come una vera e propria «lotteria» (D'Alimonte 2007; 2014) e poteva fornire esiti diversi rispetto alla Camera, dove un vincitore chiaro c'era sempre e comunque, pur in presenza di minime differenze di voto. Così fu nel 2006, quando l'Unione di Prodi ottenne una solida maggioranza alla Camera e una maggioranza invece risicatissima al Senato. E così è

\footnotetext{
${ }^{7} \mathrm{Nel} 1993$ aveva fatto la sua prima riapparizione dal 1953 il meccanismo del premio di maggioranza, previsto dalla legge 81/1993, meglio nota come legge Ciaffi, che riguardava l'elezione degli organi comunali e provinciali. Ad essa seguì due anni dopo la legge 43/1995, nota come legge Tatarella, che estendeva l'applicazione del premio di maggioranza (seppure all'interno di un sistema diverso da quelli comunale e provinciale) alle elezioni regionali. Con la legge costituzionale 1/1999 fu introdotta l'elezione diretta del presidente della giunta regionale e fu concesso alle regioni italiane il potere di scegliersi la propria legge elettorale: da allora abbiamo assistito a numerose riforme, ma il premio di maggioranza è rimasto pressoché ovunque (Chiaramonte 2007).
} 
stato, ancor più, nel 2013 quando la coalizione di centrosinistra vincitrice alla Camera è rimasta ben al di sotto della maggioranza assoluta di seggi al Senato.

Il secondo limite era connaturato alle modalità di attribuzione del premio di maggioranza. Quest'ultimo era assegnato alla lista o coalizione con più voti a prescindere dai voti da essa conseguiti, anche pochi. Qualcosa di simile è successo nel 2013, quando alla Camera la coalizione di centro-sinistra ottenne il 55\% dei seggi con meno del $30 \%$ dei voti. E se non ne è derivata una maggioranza parlamentare in grado a sé stante di formare un governo e persino di eleggere il presidente della Repubblica è proprio perché al Senato non c'è stato vincitore. Sia chiaro, livelli di sovra-rappresentazione analoghi sono presenti anche in altri contesti democratici con altri sistemi elettorali, ma in Italia, dato il nostro modello costituzionale, una distorsione troppo forte tra voti e seggi rischia di alterare gli equilibri del sistema istituzionale, modificando il ruolo del presidente della Repubblica e quindi il rapporto tra i poteri dello Stato.

Il terzo limite era connesso al modello di competizione compatibile con il sistema di soglie e di incentivi all'aggregazione. Con la legge Mattarella, grazie al collegio uninominale, lo spazio di rappresentanza dei «terzi poli» era alquanto limitato, se non nel caso di formazioni con un insediamento territoriale concentrato (come la Lega Nord nel 1996); con la legge Calderoli, invece, i «terzi poli» - anche molto piccoli - potevano contare su un trattamento analogo al «secondo polo», dal momento che i seggi residui rispetto alla quota-premio erano tutti distribuiti proporzionalmente. In altri termini, rispetto alla legge Mattarella la legge Calderoli riduceva i costi della defezione dalle due grandi coalizioni e i costi di entrata nella competizione di nuove «terze» forze, con ciò indebolendo la cogenza degli incentivi istituzionali al bipolarismo. In un primo tempo, sorretto dal consenso popolare, l'assetto bipolare non ne ha risentito - ed anzi, nel 2006, ha raggiunto l'apogeo - ma in un secondo tempo, anche grazie proprio a quel sistema elettorale, le «terze forze» politiche hanno potuto godere di uno spazio di azione sempre maggiore fino a scardinarlo del tutto.

Il quarto limite concerneva le modalità di individuazione degli eletti al parlamento. Nel clima di crescente insofferenza nei confronti della classe politica era prevedibile che la lista bloccata (dunque l'impossibilità per gli elettori di esprimere una indicazione di preferenza sui candidati) divenisse il simbolo dell'autoreferenzialità dei partiti. Alla legge Calderoli si rimproverava cioè di essere strumento delle oligarchie partitiche e di perpetuare le rispettive rendite di posizione, consentendo loro un forte controllo nella selezione dei rappresentanti in parlamento.
I limiti della legge Calderoli sin qui descritti, e se ne sono menzionati solo i principali, erano noti già da tempo e comunque ben prima delle elezioni del 2013 quando si sono manifestati nella loro pienezza. A dirla tutta, non sono mancati i tentativi di modificare questo sistema elettorale già dopo la sua prima applicazione (a cavallo tra la fine del 2007 e l'inizio del 2008) e poi anche successivamente (ad esempio nell'autunno del 2012). A più riprese sono state elaborate varie ipotesi di riforma che però non hanno fatto molta strada. Né ha avuto migliore fortuna un tentativo di modifica per via referendaria volto ad abrogare la possibilità di collegamento tra liste. L'intento era quello di attribuire il premio alla sola lista più votata, così da prevenire $\mathrm{o}$ attenuare il potere $\mathrm{di}$ ricatto dei piccoli partiti. In ogni caso, il referendum, che si svolse nel giugno 2009, non risultò valido, ancora una volta per il mancato raggiungimento del quorum di votanti. Così, l'eliminazione della legge Calderoli come fino ad allora applicata non è passata né dai partiti (via parlamento), né dagli elettori (via referendum), ma ha avuto luogo per via giudiziaria in seguito a una sentenza della Corte costituzionale nel 2014.

\section{LA VIA GIUDIZIARIA ALLE RIFORME ELETTORALI: LE SENTENZE DELLA CORTE COSTITUZIONALE} (E L'ITALICUM)

Con la sentenza 1/2014 la Corte costituzionale ha dichiarato illegittima la legge Calderoli in alcuni suoi elementi caratterizzanti, quali il premio di maggioranza e la lista bloccata. Più specificamente, il premio di maggioranza è stato dichiarato incostituzionale non in sé, ma come applicato dalla legge Calderoli, sia alla Camera sia al Senato, ossia senza previsione di una soglia minima di voti o di seggi ai fini della sua attribuzione, configurandosi così come un meccanismo premiale «foriero di una eccessiva sovra-rappresentazione della lista di maggioranza relativa, in quanto consente ad una lista che abbia ottenuto un numero di voti, anche relativamente esiguo, di acquistare la maggioranza assoluta dei voti». In modo analogo, la lista bloccata è stata dichiarata incostituzionale non in sé, ma in quanto associata (come in effetti era nella legge Calderoli) ad un elenco di candidati molto ampio che rende impossibile la loro «conoscibilità» da parte degli elettori.

Questa sentenza ha costituito di fatto una nuova riforma elettorale - la terza dopo quelle del 1993 e del 2005 - poiché il sistema di voto risultante dalla «mannaia» della Consulta era comunque applicabile (e non poteva essere diversamente). Nasceva così il consultellum, un sistema elettorale a quel punto divenuto «solo» 
proporzionale (ossia senza premio di maggioranza), che peraltro manteneva alcuni caratteri del vecchio sistema per quanto ora non più funzionali: ad esempio la possibilità di formare coalizioni (a che pro, tenuto conto della eliminazione del premio di maggioranza?) e soglie di sbarramento differenziate tra le liste unite in coalizione e le liste concorrenti da sole (che, in regime proporzionale, diventava norma a sua volta di dubbia costituzionalità) ${ }^{8}$. Non altrettanto per quanto riguardava le modalità di individuazione degli eletti, poiché la sentenza 1/2014 introduceva - attraverso un intervento a dir poco creativo - il voto di preferenza (unico) in sostituzione della lista bloccata.

La riforma elettorale per via giudiziaria, per quanto discutibile, per un po' sembrò essere un elemento di sprone per una nuova riforma elettorale "politica» e dunque un fatto temporaneo e senza effetti duraturi. Originata, non formalmente ma sostanzialmente sì, dall'inazione di un parlamento incapace di trovare un accordo su modifiche o alternative alla legge Calderoli, tale riforma sarebbe stata superata - si pensava - da una nuova iniziativa politica, che avrebbe preso forma in virtù della pressione esercitata dai presidenti Napolitano prima e Mattarella dopo, oltre che della verve del nuovo presidente del consiglio Renzi.

In effetti, il governo Renzi dette un impulso forte alla prospettiva delle riforme istituzionali, promuovendo un disegno ampio di revisione costituzionale e di nuovo sistema elettorale ad essa organicamente collegato. In accordo con la sua base parlamentare, inizialmente allargata a Forza Italia di Berlusconi, Renzi decise di procedere alla riforma elettorale prima che quella costituzionale completasse il suo percorso (del resto molto più lungo $\mathrm{e}$, prevedibilmente, anche più contrastato) e di farla con riferimento alla sola Camera dei deputati, dal momento che il progetto di revisione costituzionale prevedeva un Senato non elettivo. Al termine di una fase di intensa discussione e di progressivo affinamento, il parlamento approvò dunque la legge 52/2015, prontamente ribattezzata italicum. Si trattava della quarta riforma elettorale nazionale nel giro di poco più di venti anni.

L'italicum era ancora una volta un sistema elettorale misto, del tipo proporzionale a premio di maggioranza. Come la legge Calderoli della Camera era un sistema majority-assuring, ma diversamente da quella prevedeva

\footnotetext{
${ }^{8}$ Il sistema elettorale del Senato derivante dalla sentenza 1/2014 presenta un elemento di contraddizione nella misura in cui da un lato si configura «in entrata» come un sistema elettorale esclusivamente proporzionale e dall'altro è, «in uscita», in grado di generare livelli di sovra- e sotto-rappresentazione dei partiti paragonabili a quelli dei sistemi maggioritari, per via della ripartizione regionale dei seggi, ma soprattutto di soglie di sbarramento alquanto elevate particolarmente per le liste «solitarie».
}

una soglia del $40 \%$ dei voti ai fini dell'attribuzione del premio e il divieto di coalizione. Qualora nessuna lista avesse raggiunto tale soglia, si sarebbe svolto un secondo turno di votazione - un ballottaggio - tra le due liste con più voti al primo turno e quella vincente avrebbe ottenuto il premio di maggioranza (340 seggi). Le altre liste, purché sopra lo sbarramento del $3 \%$ dei voti, si sarebbero poi suddivise proporzionalmente i seggi restanti.

Il destino dell'italicum è stato lo stesso della riforma costituzionale. Quest'ultima, completato il percorso di approvazione parlamentare nell'aprile 2016, è stata respinta nel referendum confermativo svoltosi nel dicembre di quello stesso anno. Analogamente, l'italicum è caduto sotto la scure della Corte costituzionale, che con la sentenza 35/2017 ha dichiarato illegittime le disposizioni ivi previste relative al turno di ballottaggio per l'attribuzione del premio di maggioranza. Tuttavia, come nel caso della sentenza 1/2014 sulla legge Calderoli, anche la sentenza 35/2017 sull'italicum ha fatto in modo che le norme residuali, rispetto a quelle censurate, fossero autoapplicative. Ne è scaturito pertanto un nuovo sistema elettorale - il consultellum II - valido per la sola Camera dei deputati, che si configura ancora come un sistema proporzionale a premio di maggioranza ma che non è più majority-assuring (come era invece l'italicum). Il premio di maggioranza, infatti, è «eventuale», in quanto attribuito alla lista più votata solo nel caso in cui questa ottenga il $40 \%$ dei voti nell'unico turno di votazione ${ }^{9}$.

Con il consultellum II si è arrivati alla quinta riforma elettorale dal 1993, la terza nel giro di poco più di tre anni, la seconda per via «giudiziaria» dopo quella del 2014. Ma già una sesta riforma andava profilandosi, richiesta da più parti - in primis dal presidente della Repubblica Mattarella - e volta come minimo ad armonizzare due sistemi elettorali alquanto diversi (consultellum II per la Camera e consultellum I per il Senato, entrambi dunque derivanti da pronunce della Corte) che, se applicati nelle successive elezioni, avrebbero potuto generare esiti elettorali profondamente incoerenti tra i due rami del parlamento ${ }^{10}$.

\footnotetext{
${ }^{9}$ Il sistema elettorale della Camera derivante dalla sentenza 35/2017 si configura «in entrata» come un sistema elettorale misto, ma, «in uscita», nella sua concreta applicazione, può produrre due esiti profondamente diversi e per così dire opposti: un esito decisamente «maggioritario» qualora scatti il premio di maggioranza, ovvero un esito sostanzialmente "proporzionale» qualora il premio non scatti. Se la ratio di questo sistema può apparire comprensibile alla luce della sentenza 1/2014 della Corte costituzionale, che ha espressamente condizionato l'attribuzione di un premio di maggioranza al raggiungimento di una soglia «ragionevole», nondimeno vi è un notevole scarto tra i possibili esiti che dalla sua applicazione possono derivare.

${ }^{10}$ Le differenze tra questi due sistemi elettorali appena descritti sono numerose e profonde. Eccole in sintesi: a) la presenza del premio di
} 


\section{IL RITORNO AL COLLEGIO UNINOMINALE: LA RIFORMA DEL 2017}

Nell'ottobre 2017, appena quattro mesi prima della fine della legislatura, il Parlamento ha approvato, con il concorso di tutti i principali partiti italiani ad eccezione del M5s, l'ennesima riforma elettorale, la sesta dal 1993. La nuova legge, n. 165/2017, è stata subito ribattezzata rosatellum, dal nome di Ettore Rosato, esponente del Pd e primo firmatario. I nuovi sistemi elettorali di Camera e Senato sono di tipo misto, ma la loro componente maggioritaria non consiste più nel premio di maggioranza come era stato con la legge Calderoli e con l'italicum bensì nei collegi uninominali - che dunque fanno il loro ritorno dopo essere stati cancellati con l'abolizione della legge Mattarella nel 2005 (Chiaramonte, D’Alimonte e Paparo 2019; Pinto, Pedrazzani e Baldini 2018).

Dopo la sostanziale proporzionalizzazione apportata dalle sentenze della Corte, la riforma elettorale del 2017 è stata dunque funzionale a restituire ai sistemi elettorali di Camera e Senato una «dose» di maggioritario, peraltro molto minore di quanto non fosse con la legge Mattarella.

La legge Rosato prevede l'assegnazione di poco più di un terzo dei seggi totali (232 su 630, pari al 36,8\% alla Camera; 116 su 315 al Senato) in collegi uninominali con formula plurality. I restanti seggi sono ripartiti, a livello nazionale alla Camera e a livello regionale al Senato, tra le coalizioni e le liste individuali (non coalizzate) in base al metodo del quoziente naturale e dei più alti resti. Sono previste delle soglie di sbarramento, del $10 \%$ e del $3 \%$ su base nazionale rispettivamente per le coalizioni di liste e le liste individuali. Al Senato, partecipano alla ripartizione dei seggi anche le liste che nella rispettiva regione abbiano ottenuto almeno il $20 \%$ dei voti. Inoltre, al totale nazionale o regionale di voti delle coalizio-

maggioranza alla Camera e la sua assenza al Senato; b) la possibilità di costituire coalizioni tra liste al Senato ma non alla Camera; c) la soglia di sbarramento variabile tra liste coalizzate o meno al Senato e fissa alla Camera; d) il livello di ripartizione dei seggi tra le liste/ coalizioni, nazionale alla Camera e regionale al Senato; e) la presenza di capilista bloccati alla Camera ma non al Senato; f) il voto di preferenza unico al Senato e la doppia preferenza di genere alla Camera; g) la previsione di norme volte a favorire la rappresentanza di genere molto più stringenti alla Camera rispetto al Senato. Ad esse si sommano poi quelle già note, derivanti dai vincoli costituzionali, relativamente all'elettorato attivo e passivo, alle modalità di determinazione dei seggi spettanti alle circoscrizioni (in particolare per le regioni al Senato) e alla disposizione dell'art. 57 della Costituzione laddove recita che il Senato è eletto "su base regionale» e che è stata sin qui interpretata nel senso di impedire ladozione di meccanismi che agissero a livello sovraregionale (quali un premio di maggioranza o soglie nazionali). Che da tutte queste differenze (qualora i sistemi in questione siano applicati simultaneamente) possano scaturire esiti elettorali diversi tra i due rami del parlamento è molto probabile. ni non contribuiscono le liste che ne fanno parte ma che hanno ottenuto meno dell' $1 \%{ }^{11}$.

Le componenti maggioritaria e proporzionale del sistema elettorale non sono del tutto indipendenti l'una dall'altra. Infatti, i candidati nei collegi uninominali sono sostenuti da una o più liste concorrenti nel proporzionale. Gli elettori esprimono un voto "congiunto»: il voto per una lista si trasferisce automaticamente al candidato di collegio cui essa è collegata e viceversa. Nel caso di coalizioni partitiche a supporto del candidato di collegio, il voto espresso solo nei confronti di quest'ultimo è ripartito pro-quota tra tutte le liste della coalizione, in proporzione ai voti che tali liste ricevono nel collegio. In ogni caso, gli elettori non possono «disgiungere» il loro voto, assegnandolo ad un candidato di collegio e ad una lista non collegati tra loro.

Gli effetti esercitati dal nuovo sistema elettorale alla sua prima applicazione nelle elezioni politiche del 2018 restituiscono un'immagine composita. Da un lato, se guardiamo alla disproporzionalità della rappresentanza partitica, si trae l'impressione che esso sia stato più proporzionale che maggioritario. Dall'altro lato, se guardiamo invece al comportamento di voto e, soprattutto, al modello di competizione sembra invece prevalere l'impressione opposta. Infatti, la presenza pur numericamente ridotta di collegi uninominali si è rivelata un incentivo sufficiente per spingere i partiti a fare accordi prima del voto, ossia a coordinarsi formando coalizioni pre-elettorali. Queste coalizioni hanno quindi plasmato la competizione elettorale conferendogli un'impronta maggioritaria. In altri termini, gli elettori hanno votato non solo per un partito, come avrebbero fatto in un'arena puramente proporzionale, ma anche per una compagine candidata al governo, poiché hanno percepito le due coalizioni di centro-sinistra e di centro-destra, oltre che il Movimento 5 stelle, come vere e proprie alternative per la guida del paese.

L'assenza di un chiaro vincitore, come già era stato con la legge Calderoli nel 2013, ha infine riaperto il dibattito sulla riforma elettorale, contrapponendo ancora una volta i fautori di soluzioni più maggioritarie a quelli di soluzioni più proporzionalistiche. Tra i primi si è schierata in questa circostanza la Lega, che - forte del crescente consenso nel paese - ha addirittura promosso un referendum (ritenuto poi inammissibile dalla Corte costituzionale) per l'abolizione della quota proporzionale del rosa-

${ }^{11}$ I seggi assegnati alle liste a livello nazionale sono poi attribuiti alle stesse prima in circoscrizioni e poi in collegi plurinominali secondo una procedura volta far sì che a ciascun collegio plurinominale spetti in ultima istanza un numero di seggi pari a quello determinato ex-ante in base alla popolazione. Per ciascuna lista, i candidati sono eletti nell'ordine di presentazione, poiché le liste sono «bloccate» e non è previsto alcun voto di preferenza. 
tellum e dunque per la sua trasformazione in un sistema integralmente maggioritario all'inglese. Tra i secondi si sono invece schierati i partiti facenti parte del governo Conte II, che non a caso hanno presentato un disegno di legge per una riforma elettorale in senso proporzionale. Come che sia, anche la legge Rosato non pare destinata a durare a lungo e un'ennesima riforma è alle viste.

\section{LE RIFORME ELETTORALI IN ITALIA: UNO SGUARDO D'INSIEME}

Nei paragrafi precedenti abbiamo analizzato il contesto politico nel quale le varie riforme hanno avuto luogo, le principali proprietà dei sistemi elettorali di volta in volta introdotti e le criticità legate alla loro applicazione concreta o presunta. Sulla base delle considerazioni sviluppate possiamo tentare ora di caratterizzare nel loro insieme le riforme elettorali di questi ultimi anni. Lo facciamo, senza pretesa di costruire una teoria generale, seguendo lo schema suggerito da Benoit (2007) e dunque evidenziando riassuntivamente 1 ) chi ha deciso le riforme, ossia gli attori del cambiamento, 2) sulla base di quali preferenze e in vista di quali obbiettivi, e 3) nel contesto di quali vincoli normativi e costituzionali.

Per quanto riguarda il primo aspetto, va rilevato che le sei riforme elettorali che si sono susseguite dal 1993 ad oggi sono state il prodotto di processi diversi che hanno coinvolto una pluralità di attori. Il parlamento e, in esso, i partiti non hanno sempre mantenuto il pieno controllo della policy di riforma e talvolta l'hanno persino subita, non essendo indifferente a ciò la loro stessa crisi di legittimità. Come già messo in evidenza, accanto agli attori più prettamente politici, hanno infatti giocato un ruolo fondamentale gli elettori attraverso lo strumento referendario (si pensi al 1993, ma anche ai tentativi andati a vuoto del 1999, 2000 e 2009) e i giudici di suprema istanza attraverso le sentenze della Corte costituzionale (2014 e 2017). Se da un lato questa circostanza indica una notevole vitalità della società civile e un controllo serrato della massima magistratura su una questione cruciale come quella delle regole di voto, dall'altro conferma la vulnerabilità dei sistemi elettorali quali strumenti di ingegneria istituzionale (in quanto soggetti a veti, modifiche e correzioni provenienti da una pluralità di attori).

Circa il secondo aspetto, ossia le preferenze e gli obbiettivi degli attori convolti nei processi di riforma, si evidenzia anche in questo caso una combinazione di ingredienti variabili in funzione degli specifici attori e dello specifico processo di riforma. Laddove i partiti, o alcuni tra loro, sono stati in grado di controllare il percorso della riforma - come nel caso della legge Calderoli e dell'italicum - ha prevalso l'obbiettivo di massimizzazione dei seggi (per sè ma anche, e soprattutto, per la propria coalizione orientata a costituire un governo ${ }^{12}$ ). Persino in questi casi, tuttavia, il set di alternative davvero percorribili non è mai stato illimitato. Al contrario, era ristretto a quelle soluzioni che, al contempo, consentissero la possibilità di trovare un compromesso all'interno della coalizione proponente, e, poiché tale coalizione era coincidente con o includeva le forze della maggioranza parlamentare, garantissero anche la sopravvivenza del governo in carica (che poi era pre-condizione per il successo della riforma stessa) ${ }^{13}$. Ancora più ristretta è stata la possibilità di scelta quando i partiti non sono stati i principali attori della riforma, come ai tempi della legge Mattarella o della legge Rosato. Nel primo caso, gli spazi di manovra dei partiti erano ridottissimi per via del vincolo referendario e furono sfruttati per interventi al margine (scorporo, doppia scheda e doppio voto) sul sistema elettorale della Camera. $\mathrm{Nel}$ secondo caso, vi era un più ampio spazio di azione, ma dovendo comunque e innanzi tutto provvedere - su pressione del Presidente della Repubblica - a omogeneizzare i sistemi elettorali di Camera e Senato in vista delle elezioni e con un accordo ampio tra le forze politiche. Quando, infine, la riforma elettorale è scaturita da una sentenza della Corte costituzionale, i partiti hanno subito l'iniziativa altrui a correzione di riforme ritenute parzialmente illegittime, oltretutto dovendo poi, a propria volta, porre rimedio alle storture derivanti dal «taglia e cuci» di quegli interventi. Ne emerge, complessivamente, un quadro caratterizzato da frammentarietà di intenti e, soprattutto, fortemente condizionato dalla contingenza, sia quando si sono affermate circostanze esterne ai partiti sia quando i partiti sono riusciti a perseguire più chiaramente i propri obbiettivi.

Per quanto riguarda, infine, il contesto dei vincoli politici e costituzionali, vanno sottolineati due elementi. Il primo riguarda l'incertezza, e persino la contraddittorietà, dei limiti costituzionali in tema di riforme elettorali. La presunta piena discrezionalità del legislatore su questa materia, pur sancita dalla giurisprudenza

\footnotetext{
${ }^{12} \mathrm{Da}$ questo punto di vista si dovrebbe dunque più propriamente dire che le motivazioni della riforma elettorale non sono state solo di tipo office-seeking, ma anche di tipo policy-seeking, poiché la preferenza per un certo tipo di sistema elettorale ha tenuto conto anche delle probabilità di successo di governi diversi e dunque di policy alternative. Inoltre, incorporando aspettative circa le strategie di competizione dei partiti (specificamente: la formazione o meno di coalizioni preelettorali), questi processi di riforma sottintendono una funzione di scelta che combina elementi istituzionali e comportamentali (vedi Colomer 2004; 2018).

${ }^{13}$ Un ulteriore vincolo era poi dato dal consenso diffuso (anche nellopinione pubblica) verso sistemi elettorali che favorissero la governabilità. A lungo, dunque, un ritorno alla proporzionale pura non sarebbe stato accettabile.
} 
costituzionale, deve in realtà fare i conti con una serie di limitazioni derivanti da altrettante sentenze, non solo recenti e non solo della Corte costituzionale, che talvolta poggiano su considerazioni che rendono assai confuso il quadro di riferimento. Il secondo elemento concerne la constatazione di come, in questi ultimi venticinque anni, la legge elettorale abbia perso l'aura di «legge più importante dell'ordine costituzionale, dopo la Costituzione», come diceva Sturzo. Non è più ritenuta cioè parte integrante dell'accordo-compromesso che sta alla base del regime politico, inteso sia come insieme di norme sia come comunità di valori. Ne consegue che non costituisca più un ostacolo per un partito o per uno schieramento politico porsi nella prospettiva di approvare una riforma elettorale a maggioranza ristretta, magari, come abbiamo evidenziato in precedenza, con l'obbiettivo di ottenere un vantaggio di breve periodo per la propria parte. Cosa che poi finisce per innescare un doppio circolo vizioso. Da un lato induce altre, diverse maggioranze a fare lo stesso. Dall'altro lato, ed è la conseguenza più importante, scoraggia i partiti ad adattarsi alle nuove regole, dal momento che potranno appena possibile cambiarle. In ambedue i casi, il risultato è che si vanificano i risultati attesi dalla riforma elettorale stessa, qualunque essa sia. In altri termini, il rischio che si corre è quello di entrare in una spirale di instabilità che neutralizzi gli obbiettivi incorporati da riforme istituzionali che dovrebbero avere lungo corso per poter dispiegare i propri effetti. E che, dunque, l'instabilità porti altra instabilità e nuove soluzioni istituzionali (come i sistemi elettorali) si seguano l'una dopo l'altra alla continua e vana ricerca di un punto di equilibrio.

\section{CONCLUSIONI}

Non sono certo mancate neanche nel lungo periodo che va dall'unità d'Italia alla fine della Prima Repubblica. Ma, per numero e continuità di discussione, le riforme elettorali hanno caratterizzato soprattutto la storia degli ultimi 25-30 anni. A partire da quella del 1993, abbiamo assistito infatti a ben sei riforme elettorali, anche se non sempre i sistemi elettorali che ne sono scaturiti sono stati effettivamente applicati. E, come abbiamo visto, non è finita qua.

Certo è che, in questa girandola di riforme, che abbiamo qui esaminato nello specifico percorso che ha portato alla loro approvazione e applicazione, alcune (leggi Mattarella e Calderoli su tutte) sono state caricate di eccessive attese. A volte si è avuta l'impressione che qualcuno davvero pensasse ad esse come alla soluzione ai «mali» della politica italiana. Normale che poi tali aspettative fossero deluse, nonostante che i nuovi sistemi elettorali generassero comunque alcuni effetti (anche positivi) tra quelli realisticamente attesi. Ciò che invece è mancato e che avrebbe favorito la stabilizzazione dei nuovi sistemi elettorali sono state le tante altre riforme istituzionali che avrebbero reso il contesto della loro applicazione più favorevole e idoneo ad assecondarne gli effetti. Una di queste è la riforma del bicameralismo paritario, che avrebbe eliminato alla radice il problema delle differenze di vincoli normativi e di esiti elettorali tra Camera e Senato. Un'altra è quella della forma di governo, che sarebbe stata necessaria a sciogliere i nodi e le contraddizioni tra quanto previsto dalla lettera della Costituzione (governi a legittimazione esclusivamente parlamentare) e quanto emerso come sviluppo dell'introduzione di regole maggioritarie (governi a legittimazione anche elettorale). E poi le riforme dei regolamenti parlamentari, del finanziamento pubblico dei partiti, dell'accesso ai mezzi di comunicazione che avrebbero potuto ad esempio disinnescare l'eccesso di frammentazione partitica. Ma tutte queste riforme o non sono state fatte o sono state fatte in maniera molto parziale. Con la conseguenza che si è continuato a chiedere ad un nuovo sistema elettorale una risposta che invece doveva arrivare attraverso altri mezzi.

Forma di governo, forma di stato e sistema elettorale configurano infatti una architettura istituzionale complessa con forti legami di interdipendenza. Non si può toccarne un elemento senza tener conto degli effetti che la modifica può avere sull'equilibrio sistemico complessivo. Invece la Seconda Repubblica è nata e si è sviluppata in maniera disorganica seguendo spinte e interessi contingenti e spesso divergenti. Il risultato è un sistema squilibrato. Il rischio attuale è che invece di nuovi e più convincenti equilibri istituzionali prevalgano spinte centrifughe dirompenti. Se si vuole invertire la tendenza è quindi necessario pensare alla riforma elettorale congiuntamente ad una riforma più ampia del sistema istituzionale. Ciò richiede tuttavia un consenso di fondo su una visione condivisa di democrazia. Ed è forse la mancanza di tale consenso che, in ultima istanza, spiega, nella storia d'Italia, specialmente quella più recente, la continua ricerca, quasi ossessiva ormai, di nuove soluzioni istituzionali che troppo spesso si concentrano sulle (e si limitano alle) sole riforme elettorali.

\section{RIFERIMENTI BIBLIOGRAFICI}

Baldini, G. (2011), The Different Trajectories of Italian Electoral Reforms, in «West European Politics», 34, 3, pp. 644-663. 
Benoit, K. (2007), Electoral Laws as Political Consequences: Explaining the Origins and Change of Electoral Institutions, in "Annual Review of Political Science», 10, pp. 363-390.

Cazzola, F. (1972), Partiti, correnti e voto di preferenza, in «Rivista Italiana di Scienza Politica», 2, pp. 569-588.

Chiaramonte, A. (2007), Il rendimento dei sistemi elettorali regionali: un quadro comparato, in Riforme istituzionali e rappresentanza politica nelle regioni italiane, a cura di A. Chiaramonte e G. Tarli Barbieri, Bologna, Il Mulino, pp. 221-250.

Chiaramonte, A. e D'Alimonte, R. (2006), Proporzionale ma non solo. La riforma della Casa delle libertà, in «Il Mulino», 56, pp. 34-45.

Chiaramonte, A. D'Alimonte, R. e Paparo, A (2019), Tra maggioritario e proporzionale. Gli effetti "misti" del nuovo sistema elettorale, in Il voto del cambiamento. Le elezioni politiche del 2018, a cura di A. Chiaramonte e L. De Sio, Bologna, Il Mulino, pp. 177-207.

Chiaramonte, A. e Di Virgilio, A. (2006), Da una riforma elettorale all'altra: partiti, coalizioni e processi di apprendimento, in «Rivista Italiana di Scienza Politica», 36, pp. 363-391.

Chiaramonte, A. e Tarli Barbieri, G. (a cura di) (2011), Il premio di maggioranza. Origini, applicazioni e implicazioni di una peculiarità italiana, Roma, Carocci.

Colomer, J.P (a cura di) (2004), Handbook of electoral system choice, Londra e New York, Palgrave-Macmillan.

Colomer, J.P. (2018), Party system effects on electoral systems, in a cura di E.S. Herron, R.J. Pekkanen, e M.S. Shugart, The Oxford handbook of electoral systems, Oxford, Oxford University Press, pp. 69-84.

D'Alimonte, R. (2001), Mixed Electoral Rules, Partisan Realignment, and Party System Change, in MixedMember Electoral Systems. The Best of Both Worlds?, a cura di M.S. Shugart e M. Wattemberg, Oxford, Oxford University Press, pp. 323-350.

D'Alimonte, R. (2005), Italy: A Case of Fragmented Bipolarism, in The Politics of Electoral Systems, a cura di M. Gallagher e P. Mitchell, Oxford, Oxford University Press, pp. 253-276.

D'Alimonte, R. (2007), Il nuovo sistema elettorale. Dal collegio uninominale al premio di maggioranza, in Proporzionale ma non solo. Le elezioni politiche del 2006, a cura di R. D’Alimonte e A. Chiaramonte, Bologna, Il Mulino, pp. 51-88.

D’Alimonte, R. (2014), Il Senato: una lotteria senza vincitori, in Terremoto elettorale. Le elezioni politiche del 2013, a cura di A. Chiaramonte e. L. De Sio, Bologna, Il Mulino, pp. 153-172.

D'Alimonte, R. e Chiaramonte, A. (1995), Il nuovo sistema elettorale italiano: le opportunità e le scelte, in
Maggioritario ma non troppo, a cura di S. Bartolini e R. D’Alimonte, Bologna, Il Mulino, pp. 37-81.

D’Amico, R. (1987), Voto di preferenza, movimento dell'elettorato e modelli di partito: l'andamento delle preferenze nelle elezioni politiche italiane del quindicennio 1968-1983, in "Quaderni dell'osservatorio elettorale», 18, pp. 91-147.

Di Virgilio, A. (2002), Lofferta elettorale: la politica delle alleanze si istituzionalizza, in Maggioritario finalmente? La transizione elettorale 1994-2001, a cura di R. D'Alimonte e S. Bartolini, Bologna, Il Mulino, pp. 79-129.

Di Virgilio, A. (2007a), Legge elettorale: correggere $i$ difetti per chiudere un ciclo, in «il Mulino», 57, pp. 61-71.

Di Virgilio, A. (2007b), La riforma elettorale della Casa delle libertà alla prova del voto, in «Polis», 21, pp. 119-146.

Donovan, M. (1995), The Politics of Electoral Reform in Italy, in "International Political Science Review / Revue internationale de science politique», 16, pp. 47-64.

Feltrini, P. e Fabrizio, D. (2007), Proporzionale con premio di maggioranza: un sistema elettorale (inconsapevolmente) efficace, in Nel segreto dell'urna. Un'analisi delle elezioni politiche del 2006, a cura di P. Feltrin, P. Natale e L. Ricolfi, Torino, Utet, pp. 323-338.

Feltrin, P. e Fabrizio, D. (2008), Il Porcellum: una legge che funziona, in Senza più sinistra. L'Italia di Bossi e Berlusconi, a cura di R. Mannheimer e P. Natale, Milano, ed. il Sole 24 Ore, pp. 145-156.

Giannetti, D. e Grofman, B. (a cura di) (2011), A Natural Experiment On Electoral Law Reform: Evaluating The Long Run Consequences Of 1990s Electoral Reform In Italy And Japan, New York, Springer.

Katz, R.S. e Bardi, L. (1979), Voto di preferenza e ricambio del personale parlamentare in Italia (1963-1976), in «Rivista Italiana di Scienza Politica», 9, pp. 71-95.

Lijphart, A. (2012), Patterns of Democracy: Government forms and Performance in Thirty-Six Countries, New Haven, Co., Yale University Press, 2 a edizione.

Pappalardo, A. (1995), La nuova legge elettorale in parlamento: chi, come e perché, in Maggioritario ma non troppo, a cura di S. Bartolini e R. D’Alimonte, Bologna, Il Mulino, pp. 13-36.

Pasquino, G. (1972), Le radici del frazionismo e il voto di preferenza, in «Rivista Italiana di Scienza Politica», 2, pp. 353-368.

Pasquino, G. (1982), Degenerazione dei partiti e riforme istituzionali, Roma-Bari, Laterza.

Pasquino, G. (1985), Restituire lo scettro al principe: proposte di riforma istituzionale, Roma-Bari, Laterza.

Pasquino, G. (1993), Votare un solo candidato. Le conseguenze politiche della preferenza unica, Bologna, Il Mulino. 
Pasquino, G. (2007), Tricks and Treats: The 2005 Italian Electoral Law and Its Consequences, in «South European Society and Politics», 12, pp. 79-93.

Pinto, A., Pedrazzani, A. e Baldini, G. (2018), Nuovo sistema elettorale e scelta dei candidati: cosa è cambiato?, in Il vicolo cieco. Le elezioni del 4 marzo 2018, a cura di M. Valbruzzi e R. Vignati, Bologna, Il Mulino, pp. 99-125.

Piretti, M.S. (2003), La legge truffa, Bologna, Il Mulino.

Quagliariello, G. (2003), La legge elettorale del 1953, Bologna, Il Mulino.

Renwick, A. (2010), The Politics of Electoral Reform. Changing the Rules of Democracy, Cambridge, Cambridge University Press.

Renwick, A., Hanretty, C. e Hine, D. (2009), Partisan selfinterest and electoral reform: the new Italian electoral law of 2005, in «Electoral studies», 28, pp. 437-447.

Ruffilli, R. (a cura di) (1987), Materiali per la riforma elettorale, Bologna, Il Mulino.

Sartori, G. (1976), Parties and Party Systems: A Framework of Analysis, New York, Cambridge University Press.

Sartori, G. (1995), Ingegneria costituzionale comparata, Bologna, Il Mulino, 1995.

Sartori, G. (2001), Il sistema elettorale resta cattivo, in «Rivista Italiana di Scienza Politica», 31, pp. 471-480.

Tarli Barbieri, G. (2018), La legislazione elettorale nellordinamento italiano, Giuffrè Francis Lefebvre, Milano.

Uleri, P.V. (2003), Referendum e democrazia. Una prospettiva comparata, Bologna, Il Mulino. 